\title{
Verzeichnis der Mitarbeiter
}

Die im folgenden aufgeführten Wissenschaftlerinnen und Wissenschaftler waren - in Zusammenarbeit mit der Wörterbuch-Redaktion des Verlages - an der Bearbeitung beteiligt.

Dipl. oec. troph. Johanne Aalderink Institut für Ernährungswissenschaft Justus-Liebig-Universität Gießen Wilhelmstraße 20 35392 Gießen

Dr. Henning Albrecht

Karl und Veronica Carstens-Stiftung Barkhovenallee 1 45239 Essen (Heidhausen)

Professor Dr. med. Malte Bühring Abteilung für Naturheilkunde im Universitätsklinikum Benjamin Franklin Turmstr. 21

10559 Berlin

Professor Dr. jur. Dr. phil. Lutz Dietze

Bergstr. 23

27726 Worpswede

Professor Dr. Dr. Klaus Dörner Westfälische Klinik für Psychiatrie, Psychosomatik und Neurologie Gütersloh Hermann-Simon-Str. 7

33334 Gütersloh

Friedhelm Eickmann

Westfälische Klinik für Psychiatrie,

Psychosomatik und Neurologie Gütersloh Hermann-Simon-Str. 7

33334 Gütersloh

\section{Professor Dr. Dr. Edzard Ernst}

University of Exeter

Centre for Complementary Health Studies

25 Victoria Park Road

Exeter EX2 4NT

Dr. med. Volker Fintelmann

Leitender Arzt der Medizinischen Abteilung B

Krankenhaus Rissen

Suurheid 20

22559 Hamburg

Dr. Otto Isaac

Liesingstraße 8

63457 Hanau
Dr. med. Iris Jiko

Westfälische Klinik für Psychiatrie, Psychosomatik und Neurologie Gütersloh Hermann-Simon-Str. 7

33334 Gütersloh

Professor Dr. med. Klaus Jork

Institut für Allgemeinmedizin

Johann Wolfgang Goethe-Universität

Theodor-Stern-Kai 7

60590 Frankfurt am Main

Daniel Kaiser

Groner Landstr. 55 c/197

37081 Göttingen

Eckard Krüger

Düsseldorfer Str. 23

45145 Essen

Professor Dr. Claus Leitzmann

Institut für Ernährungswissenschaft

Justus-Liebig-Universität Gießen

Wilhelmstraße 20

35392 Gießen

Dr. med. Thomas Lux

Senckenbergisches Institut für Geschichte der Medizin

Johann Wolfgang Goethe-Universität

Theodor-Stern-Kai 7

60590 Frankfurt

Dr. med. Dieter Melchart

Projekt „Münchener Modell“

Ludwig-Maximilians-Universität

Kaiserstr. 9

80801 München

Peter Petereit

Eichsfelderstr. 2

48153 Münster

Richard Redl

Diplom-Psychologe in Bioenergetischer

Analyse

Horst 14

48301 Nottuln 
Assessor Emil Reiling

Institut für geschichtliche Rechtswissenschaft

Universität Heidelberg

Friedrich-Ebert-Platz 2

69117 Heidelberg

Dr. med. Hans-Joachim Rudolph

Facharzt für Innere Medizin und

Rheumatologie - Physikalische Therapie,

Naturheilverfahren;

Beauftragter der Ärztekammer Berlin für Indische Medizin

Oberarzt am Wichern-Krankenhaus

Evang. Johannesstift

Schönwalder Allee 26

13587 Berlin

Dr. Barbara Schilcher

Gierkezeile 36/IV

10585 Berlin

Professor Dr. Heinz Schilcher

Institut für Pharmazeutische Biologie

Freie Universität Berlin

Königin-Luise-Str. 2 u. 4

14195 Berlin

Professor h. c. (China Medical College, Taichung, Taiwan, Rep. China)

Dr. med. Claus C. Schnorrenberger

Silberbachstraße 10

79100 Freiburg
Dr. med. Wolfgang Schulz

Westfälische Klinik für Psychiatrie,

Psychosomatik und Neurologie Gütersloh

Hermann-Simon-Str. 7

33334 Gütersloh

Dr. med. Dr. rer. nat. Bernhard Uehleke

Abtsleitenweg 11

97074 Würzburg

Theiß Urbahn

Arzt für Psychiatrie und Psychotherapie

Westfälische Klinik für Psychiatrie,

Psychosomatik und Neurologie Gütersloh

Hermann-Simon-Str. 7

33334 Gütersloh

Thorolf Weißhuhn

Landstr. $16 \mathrm{~d}$

42781 Haan/Rhld.

Martin Wollschläger

Diplompsychologe für Klinische Psychologie

und Sozialpsychiatrie

Westfälische Klinik für Psychiatrie,

Psychosomatik und Neurologie Gütersloh

Hermann-Simon-Str. 7

33334 Gütersloh

\section{Redaktion}

Dipl.-Bibl. Regina Engst

Dr. phil. Helmut Hildebrandt

Dr. med. Clemens Pätzold 

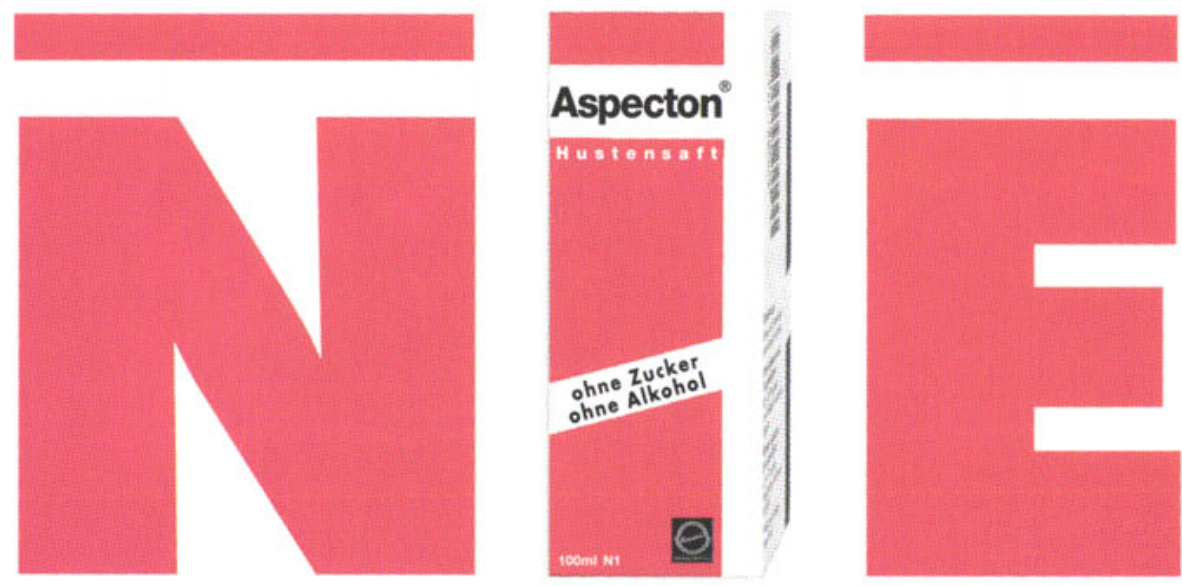

\section{ZU VIEL HUSTEN!}

\section{Aspecton ${ }^{\circledast}$ macht das schon}

So lindert Aspecton Ihre Beschwerden mit natürlichen Arzneistoffen.

Aspecton ${ }^{\oplus}$ wirkt antibakteriell und entkrampfend auf die entzündeten Luftwege. Gleichzeitig löst es den zähen Schleim und erleichtert dadurch das notwendige Abhusten, mit dem der Körper die Luftwege freihält.

Erhältlich als Saft und Tropfen.

\section{- pflanzlich}

ohne Zucker

ohne Alkohol

Aspecton ${ }^{(8)}$ Hustensaft, Wirkstoff: Thymianfluidextrakt. Aspecton ${ }^{\circledR}$ N, Hustentropfen. Anwendungsgebiete: Krankheitszeichen der Entzündung der Atemwege und Entzündung der oberen Luftwege mit Schleimbildung. Nebenwirkungen: Keine bekannt (Aspecton® Hustensaff). In seltenen Fällen Magenschleimhautreizun gen (Aspecton ${ }^{\circledR}$ N). Hinweis: Aspecton ${ }^{\circledR}$ N und Aspecton ${ }^{\circledR}$ Hustensaft sind zucker- und alkoholfreil Daher auch für Diabetiker und Patienten, die Alkohol vermeiden müssen, geeignet. KREWEL-WERKE GMBH, 53783 EITORF 


\section{Ursprüngliches wird moderne Therapie !}

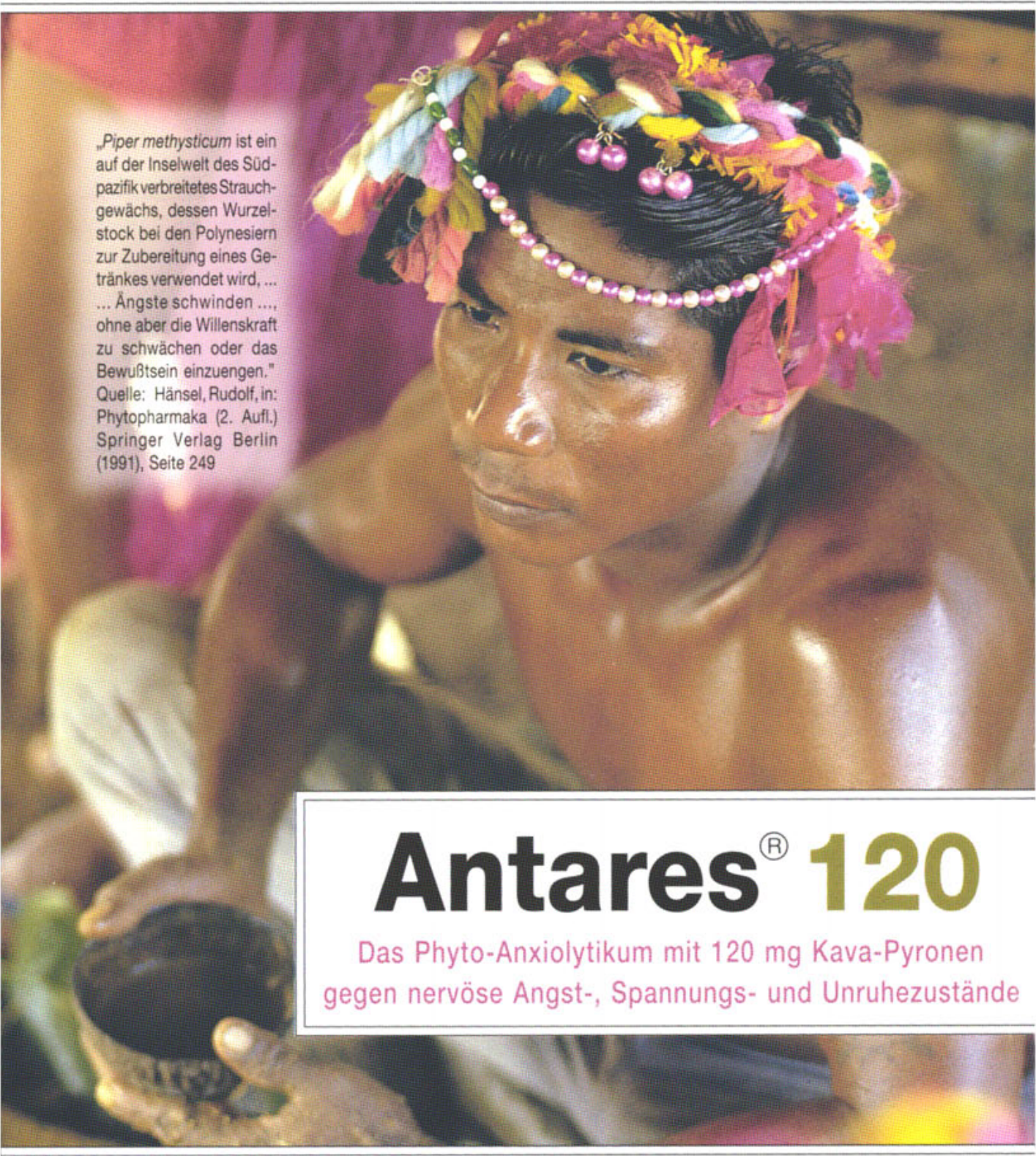

Antares 120, Wirkstoff: Kava-Kava-Extrakt. Anwendungsgebiete: Nervōse Angst-, Spannungs- und Unruhezustände. Gegenanzeigen: Schwangerschaft, Stillzeit, endogene Depressionen. Nebenwirkungen: Selten Hautreaktionen auf Überempfindlichkeitsbasis, sehr selten andere Überempfindlichkeitsreaktionen und leichte Magenbeschwerden. Hinweis: Bei länger dauernder Einnahme von Kava-Kava-Zubereitungen kann eine vorübergehende Gelbfärbung der Haut und Hautanhangsgebilde auftreten. Sollte dies auch bei Antares ${ }^{3} 120$ der Fall sein, so ist von einer weiteren Einnahme abzusehen. Weiterhin wurden Pupillenerweiterung, Störungen des Nahsehens und der abgestimmten Bewegung der beiden Augen nach Anwendung von Kava-Kava-Zubereitungen beschrieben. Stand: 10/95 KREWEL-WERKE GMBH, 53783 Eitort

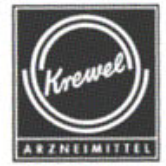

